\title{
Sharp Thresholds for Relative Neighborhood Graphs in Wireless Ad Hoc Networks
}

\author{
Chih-Wei Yi, Member, IEEE, Peng-Jun Wan, Lixin Wang, and Chao-Min Su
}

\begin{abstract}
In wireless ad hoc networks, relative neighborhood graphs (RNGs) are widely used for topology control. If every node has the same transmission radius, then an RNG can be locally constructed by using only one hop information if the transmission radius is set no less than the largest edge length of the RNG. The largest RNG edge length is called the critical transmission radius for the RNG. In this paper, we consider the RNG over a Poisson point process with mean density $n$ in a unit-area disk.
\end{abstract} Let $\beta_{0}=\sqrt{1 /\left(\frac{2}{3}-\frac{\sqrt{3}}{2 \pi}\right)} \approx 1.6$. We show that the largest RNG edge length is asymptotically almost surely at most $\beta \sqrt{\frac{\ln n}{\pi n}}$ for any fixed $\beta>\beta_{0}$ and at least $\beta \sqrt{\frac{\ln n}{\pi n}}$ for any fixed $\beta<\beta_{0}$. This implies that the threshold width of the critical transmission radius is $o\left(\sqrt{\frac{\ln n}{n}}\right)$. In addition, we also prove that for any constant $\xi$, the expected number of RNG edges whose lengths are not less than $\beta_{0} \sqrt{\frac{\ln n+\xi}{\pi n}}$ is asymptotically equal to $\frac{\beta_{0}^{2}}{2} e^{-\xi}$.

Index Terms-Wireless ad hoc networks, relative neighborhood graphs, critical transmission radii, Poisson point processes, thresholds.

\section{INTRODUCTION}

A wireless ad hoc network is a collection of radio devices located in a geographic region. Each node is equipped with an omni-directional antenna with limited transmission power. A communication session is established either through a single-hop radio transmission if the communication parties are close enough, or through relaying by intermediate devices otherwise. Because of no need for fixed infrastructures, wireless ad hoc networks can be flexibly deployed at low cost for varying missions such as battlefield decision making, emergency disaster relief and environmental monitoring.

In slow fading channel models, signals transmitted at power level $p$ will be received at distance $d$ with strength $c d^{-\kappa} p$ where $\kappa$ is an environment-related constant, called path loss factors, and $c$ is an antenna-related constant. A received signal can be decoded only if its strength is not less than a threshold. In a homogeneous network, due to the same (maximal) transmission power and similar environments, we

Manuscript received September 22, 2006; revised October 1, 2007; accepted June 7, 2009. The associate editors coordinating the review of this paper and approving it for publication were R. Negi and V. K. Bhargava.

C.-W. Yi (corresponding author) and C.-M. Su are with the Department of Computer Science, National Chiao Tung University, Hsinchu City 30010, Taiwan (e-mail: yi@cs.nctu.edu.tw, adjaumin@et4.thit.edu.tw).

P.-J. Wan and L. Wang are with the Department of Computer Science, Illinois Institute of Technology, Chicago, IL 60616 (e-mail: wan@cs.iit.edu, wanglix@iit.edu).

Part of Dr. Wan's work was conducted during his visit at the Department of Computer Science, City University of Hong Kong, Hong Kong.

Digital Object Identifier 10.1109/TWC.2010.02.060736 assume all nodes have the same (maximal) transmission radius $r$. The induced network topology is called $r$-disk graphs in which two nodes have an edge if and only if the distance between them is at most $r$. In many applications, a large number of ad hoc wireless devices are randomly deployed in a (finite) deployment region. Consequently, it is natural to represent the vertex set by a random point process in a bounded region, and the induced $r$-disk graphs are called random geometric graphs [1] which are a bounded version of the network model proposed by Gilbert (1961) [2].

Without fixed infrastructures, virtual backbones are constructed and maintained for routing packets. In [3] and [4], relative neighborhood graphs (RNGs) [5] and Gabriel graphs (GGs) [2] are used to construct planar virtual backbones. However, most previous works assumed underlying networks are connected, or implicitly assumed the transmission radius can be arbitrarily increased if necessary. In addition, in order to locally construct virtual backbones, only part of structures will be constructed. This could increase the dilation factor and cause more energy consumption. In this work, by investigating the largest RNG edge length, we show that "complete" RNGs can be locally constructed with high probability by using only 1-hop information if the transmission radius is properly set.

A graph property is said increasing if a graph has this property, then all its supergraphs are with this property. For a given set of nodes and an increasing property, the smallest transmission radius such that the induced network topology has the specified property is called the critical transmission radius for this property. The property of an $r$-disk graph without isolated nodes is increasing, and the corresponding critical transmission radius is equal to the largest nearest-neighbor link length. The largest nearest-neighbor link problem had been studied by Dette and Henze (1989) [6]. Connectivity is another increasing property. Philips et al. (1989) [7] proved that if the average number of neighbors is less than $\beta \ln n$ for some constant $\beta<1$, the network is almost surely disconnected. So, there is no (finite) magic number for connectivity. They also conjectured that $\beta>1$ is a sufficient condition for connectivity. Penrose (1997) [8] proved that the two critical transmission radii for connectivity and for no isolated nodes are asymptotically equal. Santi (2003) [9] studied the connectivity problem on a more general network model in which nodes are deployed in a cube $[0, l]^{d}$ for $d=1,2,3$ and the transmission radius is a function of $l$ and $n$. Kozma et al. (2004) [10] proved that the largest Delaunay triangulation edge length is $O\left(\sqrt[3]{\frac{\ln n}{n}}\right)$. Goel et al. (2004) [11] showed that all monotonic properties have sharp thresholds and the threshold 


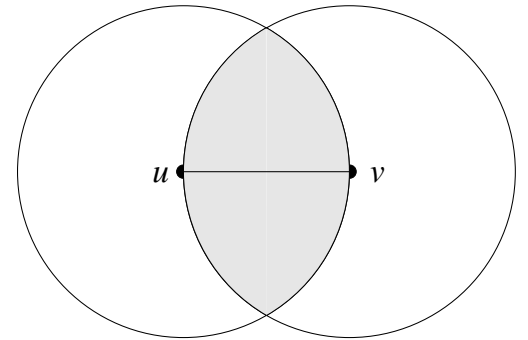

Fig. 1. The shaded lunar area in the intersection of two disks with radii $\|u-v\|$ and centered at $u$ and $v$ respectively. $u v$ is an RNG edge if and only if no other node is in the shaded area.

width is upper bounded by $O\left(\frac{\ln ^{3 / 4} n}{\sqrt{n}}\right)$ in the plane.

In RNGs, two nodes have an edge if and only if there are no other nodes in the intersection of two disks centered respectively at the two nodes and with the distance between them as radii. In Fig. 1, $u$ and $v$ have an RNG edge between them if and only if the shaded area, called a lens, doesn't contain any other node. Assume all nodes have the same transmission radius $r$. If $r$ is no less than the largest RNG edge length, the RNG is a subgraph of the $r$-disk graph. So, only edges of the $r$-disk graph are candidates of RNG edges. For any two neighboring nodes, all nodes in the corresponding lens are neighbors of these two nodes. Thus, 1-hop information is enough to decide RNG edges. On the other hand, if $r$ is less than the largest RNG edge length, it is easy to construct a counter example in which RNG edge can not be decided according to only 1-hop information. Hence, the largest edge length of an RNG is the critical transmission radius to construct the RNG by using only 1-hop information.

In this paper, we assume a wireless ad hoc network is represented by a Poisson point process in a unit-area disk with mean density $n$, denoted by $\mathcal{P}_{n}$, and all nodes have the same maximal transmission radius $r_{n}$ which is a function of $n$. We use $\mathcal{G}_{n}$ to denote the RNG over $\mathcal{P}_{n}$ and $\lambda_{n}$ to denote the maximal edge length of $\mathcal{G}_{n}$. Let $\beta_{0}=\sqrt{1 /\left(\frac{2}{3}-\frac{\sqrt{3}}{2 \pi}\right)}$. We prove that for any constant $\beta, \lambda_{n}$ is asymptotically almost surely at most $\beta \sqrt{\frac{\ln n}{\pi n}}$ if $\beta>\beta_{0}$, and at least $\beta \sqrt{\frac{\ln n}{\pi n}}$ if $\beta<\beta_{0}$. This result implies that the threshold width of the critical transmission radius is $o\left(\sqrt{\frac{\ln n}{n}}\right)$. Furthermore, let $l_{n}=\beta_{0} \sqrt{\frac{\ln n+\xi}{\pi n}}$ for some constant $\xi$ and $\mathcal{N}_{n}$ denote the number of RNG edges whose lengths are at least $l_{n}$. For convenience, these edges are called long (RNG) edges. We prove that the expected number of long RNG edges is asymptotically equal to $\frac{\beta_{0}{ }^{2}}{2} e^{-\xi}$.

In what follows, $\|x\|$ denotes the Euclidean norm of a point $x \in \mathbb{R}^{2} .|A|$ is shorthand for 2-dimensional Lebesgue measure (or area) of a measurable set $A \subset \mathbb{R}^{2}$. All integrals considered will be Lebesgue integrals. The topological boundary of a set $A \subset \mathbb{R}^{2}$ is denoted by $\partial A$. The disk of radius $r$ centered at $x$ is denoted by $B(x, r)$. The unit-area disk centered at the origin is denoted by $\mathbb{D}$, and $R_{0}=1 / \sqrt{\pi}$ is the radius of $\mathbb{D}$. Let $n$ denote the node density of the Poisson point process. An event is said to be asymptotic almost sure (abbreviated by a.a.s.) if it occurs with a probability converges to one as $n \rightarrow \infty$.
The symbols $O, o, \sim$ always refer to the limit $n \rightarrow \infty$. To avoid trivialities, we tacitly assume $n$ to be sufficiently large if necessary. For simplicity of notation, the dependence of sets and random variables on $n$ will be frequently suppressed.

The rest of this paper is organized as follows. In Section II, we give a brief of our main results. In Section III, we introduce some terminologies and present several useful geometric and integral lemmas. In Section IV, we derive the asymptotic length of the longest RNG edge. In Section V, we derive the asymptotic expected number of long RNG edges. We give simulation results in Section VI and summarize this paper in Section VII.

\section{MAin Results}

Recall that $\mathcal{P}_{n}$ denotes a Poisson point process in a unit-area disk with mean density $n, \mathcal{G}_{n}$ denotes the RNG over $\mathcal{P}_{n}, \lambda_{n}$ denotes the largest edge length of $\mathcal{G}_{n}$, and $\beta_{0}=\sqrt{1 /\left(\frac{2}{3}-\frac{\sqrt{3}}{2 \pi}\right)} \approx 1.6$. One of our main results is the following theorem.

Theorem 1: For any constant $\varepsilon>0$, as $n \rightarrow \infty$, we have

$$
\operatorname{Pr}\left[(1-\varepsilon) \beta_{0} \sqrt{\frac{\ln n}{\pi n}} \leq \lambda_{n} \leq(1+\varepsilon) \beta_{0} \sqrt{\frac{\ln n}{\pi n}}\right] \rightarrow 1 .
$$

Let $r_{n}=\beta \sqrt{\frac{\ln n}{\pi n}}$. According to Theorem 1, the $r_{n}$-disk graph over $\mathcal{P}_{n}$ a.a.s. contains $\mathcal{G}_{n}$ if $\beta>\beta_{0}$, and on the other hand, the $r_{n}$-disk graph a.a.s. does not contain $\mathcal{G}_{n}$ if $\beta<\beta_{0}$. Therefore, $\beta_{0}$ is corresponding to the threshold of the critical transmission radius for RNGs. For reference, we remark that $\beta=1$ is corresponding to the threshold for connectivity [12] [13]. The threshold width of RNGs implied by Theorem 1 is $o\left(\sqrt{\frac{\ln n}{n}}\right)$ that is far smaller than $O\left(\frac{\ln ^{3 / 4} n}{\sqrt{n}}\right)$ given in [11].

The next theorem gives the asymptotic expected number of long RNG edges. Recall that $l_{n}=\beta_{0} \sqrt{\frac{\ln n+\xi}{\pi n}}$ for some constant $\xi$, and $\mathcal{N}_{n}$ denotes the number of long RNG edges whose lengths are not less than $l_{n}$.

Theorem 2: For the expected number of long RNG edges, we have

$$
\lim _{n \rightarrow \infty} \mathbf{E}\left[\mathcal{N}_{n}\right]=\frac{\beta_{0}^{2}}{2} e^{-\xi} .
$$

Since $\operatorname{Pr}[X=0]=1-\operatorname{Pr}[X \geq 1] \geq 1-\mathbf{E}[X]$ for any non-negative integer value RV $X$,

$$
\begin{aligned}
\operatorname{Pr}\left[\lambda_{n}<\beta_{0} \sqrt{\frac{\ln n+\xi}{\pi n}}\right] & =\operatorname{Pr}\left[\mathcal{N}_{n}=0\right] \\
& \geq 1-\mathbf{E}\left[\mathcal{N}_{n}\right] \\
& \sim 1-\frac{\beta_{0}{ }^{2}}{2} e^{-\xi} .
\end{aligned}
$$

Therefore,

$$
\lim _{\xi \rightarrow \infty} \lim _{n \rightarrow \infty} \operatorname{Pr}\left[\lambda_{n}<\beta_{0} \sqrt{\frac{\ln n+\xi}{\pi n}}\right]=1,
$$

and thus $\xi \rightarrow \infty$ is an a.a.s. sufficient condition for $\lambda_{n}<$ $\beta_{0} \sqrt{\frac{\ln n+\xi}{\pi n}}$. 


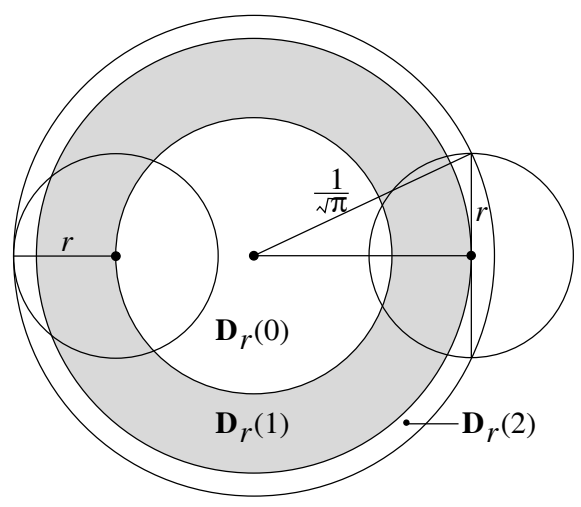

Fig. 2. The partition of the unit-area disk $\mathbb{D}$.

\section{Preliminaries}

In this section, we introduce some terminologies and lemmas that will be used to prove our main results. For a given real number $r \in\left(0, R_{0}\right)$, the unit-area disk $\mathbb{D}$ is partitioned into $\mathbb{D}_{r}(0), \mathbb{D}_{r}(1)$ and $\mathbb{D}_{r}(2)$ as shown in Fig. $2: \mathbb{D}_{r}(0)$ is the disk of radius $1 / \sqrt{\pi}-r$ centered at the origin; $\mathbb{D}_{r}(1)$ is the annulus of radii $1 / \sqrt{\pi}-r$ and $\sqrt{1 / \pi-r^{2}}$ centered at the origin; and $\mathbb{D}_{r}(2)$ is the annulus of radii $\sqrt{1 / \pi-r^{2}}$ and $1 / \sqrt{\pi}$ centered at the origin. Then,

$$
\begin{aligned}
& \left|\mathbb{D}_{r}(0)\right|=(1-\sqrt{\pi} r)^{2}, \\
& \left|\mathbb{D}_{r}(1)\right|=2 \pi r(1 / \sqrt{\pi}-r), \\
& \left|\mathbb{D}_{r}(2)\right|=\pi r^{2} .
\end{aligned}
$$

For any two points $u$ and $v$, let $L_{u v}$ denote the lens $L_{u v}=$ $B(u,\|u-v\|) \cap B(v,\|u-v\|)$, i.e. the intersection of two disks centered at $u$ and $v$, respectively, and with radii $\|u-v\|$. We have

$$
\left|L_{u v}\right|=\frac{1}{\beta_{0}^{2}} \pi\|u-v\|^{2}
$$

where $\beta_{0}=\sqrt{1 /\left(\frac{2}{3}-\frac{\sqrt{3}}{2 \pi}\right)}$. For a lens $L_{u v}$, the middle point of $u$ and $v$ is called its center, the line segment $u v$ is called its waist, and the two intersection points of $\partial B(u,\|u-v\|)$ and $\partial B(v,\|u-v\|)$ are called its vertices. A lens is called an $r$ lens if the length of its waist is $2 r$. A lens is feasible if its waist is contained in $\mathbb{D}$. In what follows, we only consider feasible lenses. Assume $\|u-v\|=2 r$. Then, if the center of $L_{u v}$ is in $\mathbb{D}_{\sqrt{3} r}(0)$, the lens is contained in $\mathbb{D}$ and $\left|L_{u v} \cap \mathbb{D}\right|=\left|L_{u v}\right|$; if the center of $L_{u v}$ is in $\mathbb{D} \backslash \mathbb{D}_{r}(2)$, at least a half lens is contained in $\mathbb{D}$ and $\left|L_{u v} \cap \mathbb{D}\right| \geq \frac{1}{2}\left|L_{u v}\right|$. The next lemma giving a lower bound for the area of the union of two lenses is from [14] (Lemma 2).

Lemma 3: Assume $c=0.039, R>0$, and $a_{1}, b_{1}, a_{2}, b_{2} \in$ $\mathbb{R}^{2}$. Let $z_{1}=\frac{1}{2}\left(a_{1}+b_{1}\right), r_{1}=\left\|a_{1}-b_{1}\right\|, z_{2}=\frac{1}{2}\left(a_{2}+b_{2}\right)$, and $r_{2}=\left\|a_{2}-b_{2}\right\|$. If $r_{1}, r_{2} \in\left[\frac{1}{2} R, R\right],\left\|z_{1}-z_{2}\right\| \leq \sqrt{3} R$, $a_{1}, b_{1} \notin L_{a_{2} b_{2}}$, and $a_{2}, b_{2} \notin L_{a_{1} b_{1}}$, then

$$
\left|L_{a_{1} b_{1}} \cup L_{a_{2} b_{2}}\right|-\left|L_{a_{1} b_{1}}\right| \geq c R\left\|z_{1}-z_{2}\right\| .
$$

An $\varepsilon$-tessellation is to divide the plane by vertical and horizontal lines into grid cells with width $\varepsilon$. Without loss of generality, we assume the $x$-axis and $y$-axis are one of the horizontal lines and vertical lines, and the origin is at a

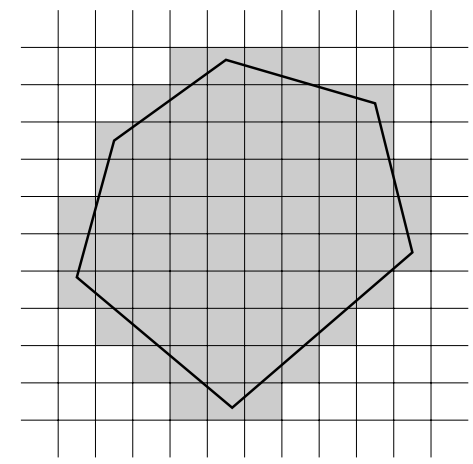

Fig. 3. A polyquadrate is a collection of grids that intersect with a convex polygon.

corner of a grid cell. A collection of grid cells intersecting with a convex polygon (or a compact convex set) is called a polyquadrate. For example, in Fig. 3, the shaded grid cells form a polyquadrate. The horizontal span of a polyquadrate is the horizontal distance measured by the number of grid cells from the leftmost cells to the rightmost cells. The vertical span of a polyquadrate is with a similar definition but for the vertical direction.

Lemma 4: If $S$ is a collection of $m$ grid cells and $n$ is a fixed positive integer, the number of polyquadrates with span less than $n$ and intersecting with $S$ is $\Theta(m)$.

Proof: Since $n$ is fixed, the number of polyquadrates that have spans less than $n$ and contain a specified grid cell is bounded. Since $S$ consists of $m$ grid cells, the lemma follows.

The next lemma gives an a.a.s. upper bound and lower bound of a collection of Poisson RVs, and we leave its proof in Appendix.

Lemma 5: Assume $c>0$ and $\beta>0$ are constant and $I_{n}=$ $\Theta\left(\left(\frac{n}{\ln n}\right)^{c}\right)$. Let $Y_{i}$ be a Poisson RV with rate $\mu_{i}$ for $i=$ $1, \cdots, I_{n}$.

1) If $\mu_{i} \geq \beta \ln n$ and $\beta \geq c$, we have

$$
\lim _{n \rightarrow \infty} \operatorname{Pr}\left[\min _{i=1}^{I_{n}} Y_{i}>0\right]=1 .
$$

2) If $Y_{1}, Y_{2}, \cdots, Y_{I_{n}}$ are independent, $\beta \in(0, c)$, and $\mu_{i} \leq$ $\beta \ln n$, we have

$$
\lim _{n \rightarrow \infty} \operatorname{Pr}\left[\min _{i=1}^{I_{n}} Y_{i}=0\right]=1 .
$$

At the end of this section, we give a lemma about the limits of integrals, and similarly, its proof can be found in Appendix.

Lemma 6: Let $r_{\xi}=\beta_{0} \sqrt{\frac{\ln n+\xi}{\pi n}}$ for some constant $\xi$, and either $R_{n}=3 \sqrt{\frac{\ln n}{\pi n}}$ or $R_{n}=\beta_{0} \sqrt{\frac{\ln n+\xi_{n}}{\pi n}}$ with $\xi_{n}=o(\ln n)$ and $\xi_{n} \rightarrow \infty$. Then

$$
\frac{n^{2}}{2} \iint_{\substack{u, v \in \mathbb{D} \\ r_{\xi} \leq\|u-v\|<R_{n}}} e^{-n\left|L_{u v} \cap \mathbb{D}\right|} d u d v \sim \frac{\beta_{0}{ }^{2}}{2} e^{-\xi} .
$$

\section{Asymptotic Length of The Longest EdGE}

This section is dedicated to the proof of Theorem 1. The proof is divided into two parts. In Subsection IV-A, we give Lemma 7 which provides an upper bound for the largest edge 
length. In Subsection IV-B, we give Lemma 8 which provides a lower bound for the largest edge length. Since two bounds are tight, Theorem 1 follows Lemma 7 and 8 .

\section{A. Upper Bound for the Longest Edge Length}

Lemma 7 says that if $\beta>\beta_{0}$, there a.a.s. do not exist RNG edges whose lengths are not less $\beta \sqrt{\frac{\ln n}{\pi n}}$. In the proof, we are going to show that any lens whose waist is fully contained in $\mathbb{D}$ and not less than $\beta \sqrt{\frac{\ln n}{\pi n}}$ a.a.s. contains some other nodes. Therefore, we can conclude that all RNG edges are a.a.s. less than $\beta \sqrt{\frac{\ln n}{\pi n}}$.

Lemma 7: For any constant $\beta>\beta_{0}$, we have

$$
\lim _{n \rightarrow \infty} \operatorname{Pr}\left[\lambda_{n} \geq \beta \sqrt{\frac{\ln n}{\pi n}}\right]=0 .
$$

Proof: Let $d=\beta \sqrt{\frac{\ln n}{\pi n}}$ and $r=\frac{d}{2}$. Pick a constant $\beta_{1} \in\left(\beta_{0}, \beta\right)$, and let $d^{\prime}=\beta_{1} \sqrt{\frac{\ln n}{\pi n}}, r^{\prime}=\frac{d^{\prime}}{2}$ and $\varepsilon=\frac{r-r^{\prime}}{\sqrt{2}}$. Let $\mathbf{C}_{r}$ be the collect of all feasible $r$-lenses, whose waists are contained in $\mathbb{D}$. If $u v$ is an RNG edge, there are no other nodes in $L_{u v}$. So, $\lambda_{n} \geq d$ implies that there exists a lens in $\mathbf{C}_{r}$ that does not contain nodes of $\mathcal{P}_{n}$. Therefore,

$$
\operatorname{Pr}\left[\lambda_{n} \geq \beta \sqrt{\frac{\ln n}{\pi n}}\right] \leq \operatorname{Pr}\left[\min _{C \in \mathbf{C}_{r}}\left|C \cap \mathcal{P}_{n}\right|=0\right] .
$$

Divide the plane by an $\varepsilon$-tessellation. The distance of any two points within a grid cell is at most $r-r^{\prime}$. If $A$ and $B$ respectively are an $r$-lens and $r^{\prime}$-lens with the same center and overlapping waists, grid cells intersected with $B$ are contained in $A$ since any point of $B$ is apart from $\partial A$ by at least $r-r^{\prime}$. So, the polyquadrate induced by $B$ is contained in $A$. Let $\left\{P_{1}, \cdots, P_{I_{n}}\right\}$ denote the set of polyquadrates induced by the $r^{\prime}$-lenses in $\mathbf{C}_{r^{\prime}}$, and $Y_{i}$ denote the number of nodes of $\mathcal{P}_{n}$ in $P_{i}$. Since any $r$-lens in $\mathbf{C}_{r}$ contains at least one $P_{i}$, we have

$$
\operatorname{Pr}\left[\min _{C \in \mathbf{C}_{r}}\left|C \cap \mathcal{P}_{n}\right|=0\right] \leq \operatorname{Pr}\left[\min _{i=1}^{I_{n}} Y_{i}=0\right] .
$$

Note that $Y_{i}$ is a Poisson RV with rate $n\left|P_{i}\right|$. Assume $E$ is the collection of polyquadrates induced by $r^{\prime}$-lenses with center on $\mathbb{D}_{\sqrt{3} r^{\prime}}(0)$ and $F$ is the collection of polyquadrates induced by feasible $r^{\prime}$-lenses with center on $\mathbb{D} \backslash \mathbb{D}_{\sqrt{3} r^{\prime}}(0)$. Since $E \cup F=\left\{P_{1}, \cdots, P_{I_{n}}\right\}$,

$$
\begin{aligned}
\operatorname{Pr}\left[\min _{i=1}^{I_{n}} Y_{i}=0\right] & =\operatorname{Pr}\left[\min _{P_{i} \in E} Y_{i}=0 \text { or } \min _{P_{i} \in F} Y_{i}=0\right] \\
& \leq \operatorname{Pr}\left[\min _{P_{i} \in E} Y_{i}=0\right]+\operatorname{Pr}\left[\min _{P_{i} \in F} Y_{i}=0\right] .
\end{aligned}
$$

For any $P_{i} \in E$, we have

$$
n\left|P_{i}\right| \geq n \frac{1}{\beta_{0}^{2}} \pi d^{\prime 2}=\left(\frac{\beta_{1}}{\beta_{0}}\right)^{2} \ln n>\ln n .
$$

Applying Lemma 4, we also have $|E|=\Theta\left(\frac{1}{\varepsilon^{2}}\right)=\Theta\left(\frac{n}{\ln n}\right)$. Therefore, by Lemma 5,

$$
\operatorname{Pr}\left[\min _{P_{i} \in E} Y_{i}=0\right]=1-\operatorname{Pr}\left[\min _{P_{i} \in E} Y_{i}>0\right] \sim 0 .
$$

For any $P_{i} \in F$, we have

$$
n\left|P_{i}\right| \geq n\left(\frac{1}{2 \beta_{0}{ }^{2}} \pi d^{\prime 2}\right)>\frac{1}{2} \ln n .
$$

Applying Lemma 4, we also have $|F|=\Theta\left(\frac{r^{\prime}}{\varepsilon^{2}}\right)=$ $\Theta\left(\sqrt{\frac{n}{\ln n}}\right)$. Therefore, by Lemma 5 ,

$$
\operatorname{Pr}\left[\min _{P_{i} \in F} Y_{i}=0\right]=1-\operatorname{Pr}\left[\min _{P_{i} \in F} Y_{i}>0\right] \sim 0 .
$$

Put Eq. 1, 2, 3, 4, and 5 together, and the lemma is proved.

\section{B. Lower Bounds for the Longest Edge Length}

Lemma 8 says that if $\beta<\beta_{0}$, there a.a.s. exist RNG edges whose lengths are not less than $\beta \sqrt{\frac{\ln n}{\pi n}}$. To prove this, the plane is tessellated into equal-size square cells. For each cell, an event that implies the existence of such RNG edges is introduced, and a lower bound for the probability of the event is derived. Since these events are identical and independent among cells, we can easily find a probability lower bound that is asymptotically equal to 1 .

Lemma 8: For any constant $\beta \in\left(0, \beta_{0}\right)$,

$$
\lim _{n \rightarrow \infty} \operatorname{Pr}\left[\lambda_{n} \geq \beta \sqrt{\frac{\ln n}{\pi n}}\right]=1 .
$$

Proof: Assume $\beta_{1}$ and $\beta_{2}$ are positive constants, and $R_{1}$ and $R_{2}$ respectively are given by $n \pi R_{1}^{2}=\beta_{1} \ln n$ and $n \pi R_{2}^{2}=\beta_{2} \ln n$. Choose $\beta_{1}, \beta_{2}$ such that $\max \left(\frac{1}{4} \beta_{0}^{2}, \beta^{2}\right)<$ $\beta_{1}<\beta_{2}<\beta_{0}^{2}$ and $\frac{\pi}{c^{2}}\left(1-\frac{R_{1}}{R_{2}}\right)<1$. Here $c$ is given by Lemma 3 . We have $\frac{1}{2} R_{2} \leq R_{1} \leq R_{2}$. Divide $\mathbb{D}$ by $\left(4 \sqrt{\frac{\ln n}{n \pi}}\right)$-tessellation. Let $I_{n}$ denote the number of grid cells fully contained in $\mathbb{D}$. Here $I_{n}=O\left(\frac{n}{\ln n}\right)$. For each such cell, we draw a disk with radius $\frac{1}{2} \sqrt{\frac{\ln n}{n \pi}}$ at the center of the cell. For $1 \leq i \leq I_{n}$, let $E_{i}$ be the event that there exist two nodes $X, Y \in \mathcal{P}_{n}$ such that $X Y$ is a RNG edge, their midpoint is in the $i$-th disk, and their distance is between $R_{1}$ and $R_{2}$. Therefore,

$$
\begin{aligned}
& \operatorname{Pr}\left[\lambda_{n} \geq \beta \sqrt{\frac{\ln n}{\pi n}}\right] \geq \operatorname{Pr}\left[\text { at least one } E_{i} \text { occur }\right] \\
&=1-\operatorname{Pr}\left[\text { none of } E_{i} \text { occurs }\right] . \\
& E_{1}, \cdots, E_{I_{i}} \text { are identical and independent. Thus, }
\end{aligned}
$$

$$
\operatorname{Pr}\left[\text { none of } E_{i} \text { occurs }\right]=\left(1-\operatorname{Pr}\left[E_{1}\right]\right)^{I_{n}} \leq e^{-I_{n} \operatorname{Pr}\left[E_{1}\right]} .
$$

We can prove $I_{n} \operatorname{Pr}\left[E_{1}\right] \rightarrow \infty$. (See Appendix B for details.) So,

$$
\operatorname{Pr}\left[\lambda_{n} \geq \beta \sqrt{\frac{\ln n}{\pi n}}\right] \rightarrow 1 .
$$




\section{EXPECTEd Number of Long EdGeS}

We have proved that the ratio of the largest RNG edge length to $\sqrt{\frac{\ln n}{n \pi}}$ is a.a.s. equal to $\beta_{0}$. Let $l_{n}=\beta_{0} \sqrt{\frac{\ln n+\xi}{n \pi}}$ for a constant $\xi$. In this section, we are going to prove Theorem 2 that gives the asymptotic expected number of long RNG edges.

Proof of Theorem 2: Assume $Y$ and $V$ are point sets and $Y \subseteq V$. Let $h_{r}(Y, V)$ denote a function such that $h_{r}(Y=\{u, v\}, V)=1$ only if $\|u-v\| \geq r$ and there is no other node of $V$ in the disk area $L_{u v}$; otherwise, $h_{r}(Y, V)=0$. Let $X_{1}$ and $X_{2}$ denote independent random points with uniform distribution over $\mathbb{D}$ and independent of $\mathcal{P}_{n}$. According to the Palm theory,

$$
\begin{aligned}
\mathbf{E}\left[\mathcal{N}_{n}\right] & =\mathbf{E}\left[\sum_{\left\{X_{1}^{\prime}, X_{2}^{\prime}\right\} \subseteq \mathcal{P}_{n}} h_{r}\left(\left\{X_{1}^{\prime}, X_{2}^{\prime}\right\}, \mathcal{P}_{n}\right)\right] \\
& =\frac{n^{2}}{2 !} \mathbf{E}\left[h_{r}\left(\left\{X_{1}, X_{2}\right\},\left\{X_{1}, X_{2}\right\} \cup \mathcal{P}_{n}\right)\right] .
\end{aligned}
$$

Let $F(r)$ be the probability of the event that $X_{1} X_{2}$ is a RNG edge and $\left\|X_{1}-X_{2}\right\| \geq r$. Then,

$$
F(r)=\mathbf{E}\left[h_{r}\left(\left\{X_{1}, X_{2}\right\},\left\{X_{1}, X_{2}\right\} \cup \mathcal{P}_{n}\right)\right],
$$

and

$$
\begin{aligned}
& F(r) \\
& =\iint_{\substack{u, v \in \mathbb{D} \\
\|u-v\| \geq r}} \operatorname{Pr}\left[\begin{array}{l|l}
X_{1} X_{2} \text { is a } & X_{1}=u \\
\mathrm{RNG} \text { edge } & X_{2}=v
\end{array}\right] d u d v \\
& =\iint_{\substack{u, v \in \mathbb{D} \\
\|u-v\| \geq r}} e^{-n\left|L_{u v} \cap \mathbb{D}\right|} d u d v .
\end{aligned}
$$

According to Theorem 1,

$$
\begin{aligned}
& F\left(\beta_{0} \sqrt{\frac{\ln n+\xi}{n \pi}}\right) \\
& \sim F\left(\beta_{0} \sqrt{\frac{\ln n+\xi}{n \pi}}\right)-F\left(3 \sqrt{\frac{\ln n}{n \pi}}\right) \\
& =\iint_{3 \sqrt{\frac{\ln n}{n \pi}}>\|u-v\| \mathbb{D} \geq \beta_{0} \sqrt{\frac{\sqrt{\ln n+\xi}}{n \pi}}} e^{-n\left|L_{u v} \cap \mathbb{D}\right|} d u d v .
\end{aligned}
$$

Hence, by Lemma 6, we have

$$
\mathbf{E}\left[\mathcal{N}_{n}\right]=\frac{n^{2}}{2 !} F\left(\beta_{0} \sqrt{\frac{\ln n+\xi}{n \pi}}\right) \sim \frac{\beta_{0}^{2}}{2} e^{-\xi} .
$$

Thus, the theorem is proved.

\section{Simulations}

We run simulations to validate our asymptotic results. In the simulation, 400 random point sets over a unit-area square with uniform distribution are generated for each node density $n=100,200,400,800$, and 1600 . The cumulative distribution functions of the largest RNG edge length are illustrated in Fig. 4. The curves from right to left are corresponding to the CDFs with respect to $n=100,200,400,800$, and 1600 . Without too much surprise, as the node density becomes larger, the largest RNG edge length becomes smaller, and the threshold

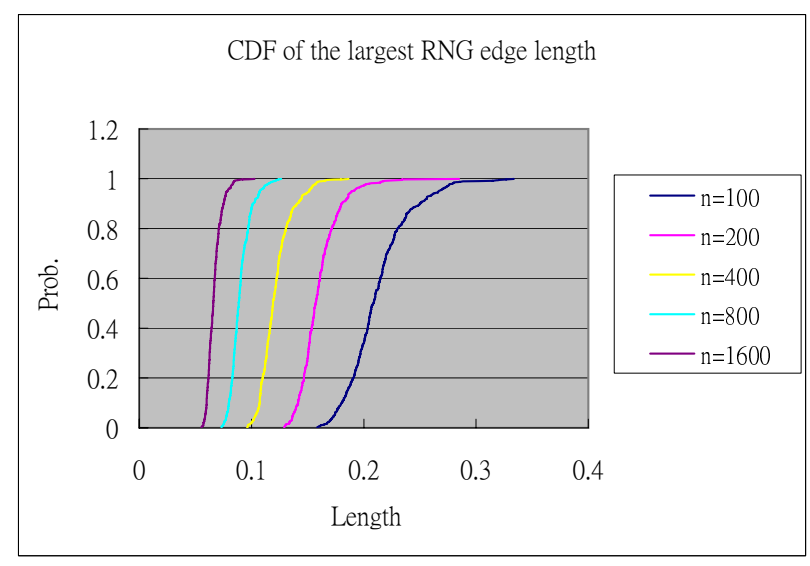

Fig. 4. CDF of the largest RNG edge length.

TABLE I

DILATION FACTORS.

\begin{tabular}{|c|c|c|c|c|c|}
\hline & $n=100$ & $n=200$ & $n=400$ & $n=800$ & $n=1600$ \\
\hline MAX & 5.039 & 6.021 & 5.795 & 5.651 & 6.977 \\
\hline MIN & 2.375 & 2.752 & 2.895 & 3.279 & 3.463 \\
\hline AVG & 3.337 & 3.659 & 3.934 & 4.177 & 4.425 \\
\hline
\end{tabular}

width, that is corresponding to the width of the CDF curve, also becomes more narrow.

To investigate the energy efficiency issue, for each pair of nodes, we calculate the ratio of the length of the shortest path in the RNG between them and the Euclidean distance between them. The maximal ratio over all pairs of nodes in a random point set is the dilation factor of the RNG. In Table I, we list the maximal, minimal, and average dilation factors among 400 random point sets for each node density. The dilation factor is a lower bound of the stretch factor. Although Bose (2002) [15] proved that the stretch factor of RNGs is $\Theta(n)$ in the worst case, we can see in Table I the dilation factor doesn't increase significantly. So, the power efficiency of RNG is not too bad in the average case.

\section{CONCLUSIONS}

The relative neighborhood graphs are a geometric structure used in topology control for wireless ad hoc networks and can be constructed by distributed and localized algorithms. Motivated by constructing geometric structures using only 1hop information, we studies the maximal length of RNG edges which is the smallest transmission radius for constructing the RNG by only 1-hop information if all nodes have the same transmission radius. In this paper, we assume a wireless ad hoc networks is represented by a Poisson point process with mean $n$ on a unit-area disk. We first showed that the ratio of the maximal length of RNG edges to $\sqrt{\frac{\ln n}{\pi n}}$ is a.a.s. equal to $\sqrt{1 /\left(\frac{2}{3}-\frac{\sqrt{3}}{2 \pi}\right)} \approx 1.6$. Next, we proved that for a constant $\xi$, the expected number of long RNG edges, whose lengths are at least $\beta_{0} \sqrt{\frac{\ln n+\xi}{\pi n}}$, are a.a.s. equal to $\frac{\beta_{0}{ }^{2}}{2} e^{-\xi}$. This imply that if $\xi \rightarrow \infty$, it is a.a.s. that the maximal length is less than $\beta_{0} \sqrt{\frac{\ln n+\xi}{\pi n}}$. 


\section{APPENDIX A \\ Proofs of LEMMAS IN SECTION III}

First, we give the proof of Lemma 5.

Proof of Lemma 5: Consider the first case. If $\mu_{i} \geq \beta \ln n$,

$$
\operatorname{Pr}\left[Y_{i}=0\right]=e^{-\mu_{i}} \leq e^{-\beta \ln n} .
$$

So,

$$
\begin{aligned}
& \operatorname{Pr}\left[\min _{i=1}^{I_{n}} Y_{i}>0\right]=1-\operatorname{Pr}\left[\exists i \text { such that } Y_{i}=0\right] \\
& =1-\operatorname{Pr}\left[\bigvee_{i=1}^{I_{n}}\left(Y_{i}=0\right)\right] \geq 1-\sum_{i=1}^{I_{n}} \operatorname{Pr}\left[Y_{i}=0\right] \\
& \geq 1-\sum_{i=1}^{I_{n}} e^{-\beta \ln n}=1-\Theta\left(\left(\frac{n}{\ln n}\right)^{c}\right) e^{-\beta \ln n} \\
& =1-\Theta\left(\frac{n^{c-\beta}}{\ln ^{c} n}\right) .
\end{aligned}
$$

Therefore, if $\beta \geq c$, we have

$$
\operatorname{Pr}\left[\min _{i=1}^{I_{n}} Y_{i}>0\right] \sim 1
$$

Consider the second case. If $Y_{1}, Y_{2}, \cdots, Y_{I_{n}}$ are independent,

$$
\begin{aligned}
& \operatorname{Pr}\left[\min _{i=1}^{I_{n}} Y_{i}>0\right]=\operatorname{Pr}\left[\bigwedge_{i=1}^{I_{n}}\left(Y_{i}>0\right)\right] \\
& =\prod_{i=1}^{I_{n}} \operatorname{Pr}\left[Y_{i}>0\right]=\prod_{i=1}^{I_{n}}\left(1-\operatorname{Pr}\left[Y_{i}=0\right]\right) \\
& \leq \prod_{i=1}^{I_{n}} e^{-\operatorname{Pr}\left[Y_{i}=0\right]}=e^{-\sum_{i=1}^{I_{n}} \operatorname{Pr}\left[Y_{i}=0\right]} .
\end{aligned}
$$

If $\mu_{i} \leq \beta \ln n$,

$$
\operatorname{Pr}\left[Y_{i}=0\right]=e^{-\mu_{i}} \geq e^{-\beta \ln n} .
$$

Put the two inequalities together. Then,

$$
\begin{aligned}
& \operatorname{Pr}\left[\min _{i=1}^{I_{n}} Y_{i}=0\right]=1-\operatorname{Pr}\left[\min _{i=1}^{I_{n}} Y_{i}>0\right] \\
& \geq 1-e^{-\sum_{i=1}^{I_{n}} \operatorname{Pr}\left[Y_{i}=0\right]} \geq 1-e^{-\sum_{i=1}^{I_{n}} e^{-\beta \ln n}} \\
& =1-e^{-\Theta\left(\left(\frac{n}{\ln n}\right)^{c}\right) e^{-\beta \ln n}}=1-e^{-\Theta\left(\frac{n^{c}-\beta}{\ln c}\right)} .
\end{aligned}
$$

Therefore, if $\beta \in(0, c)$, we have

$$
\operatorname{Pr}\left(\min _{1 \leq i \leq I_{n}} Y_{i}=0\right) \sim 1 \text {. }
$$

Next, we are going to prove Lemma 6 . But before that, we need a lemma that gives a tighter lower bound for $\left|L_{u v} \cap \mathbb{D}\right|$ as the center of $L_{u v}$ is near the boundary of $\mathbb{D}$ and a tool for variable transformation.

Lemma 9: For any $u, v \in \mathbb{D}$, let $r=\frac{1}{2}\|u-v\|, z=\frac{u+v}{2}$, and $t=\frac{1}{\sqrt{\pi}}-\|z\|$. If $z \in \mathbb{D} \backslash\left(\mathbb{D}_{\sqrt{3} r}(0) \cup \mathbb{D}_{r}(2)\right)$, we have

$$
\left|L_{u v} \cap \mathbb{D}\right| \geq \frac{1}{2}\left|L_{u v}\right|+r t .
$$

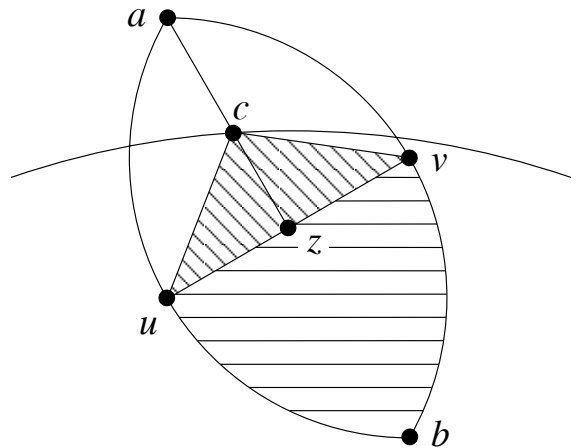

Fig. 5. A lens near the boundary of $\mathbb{D}$.

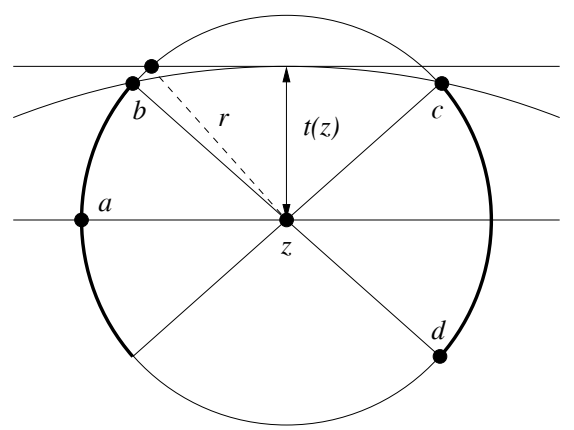

Fig. 6. If $z \in \mathbb{D}_{r}(1)$, then $\theta(z, r)=4 \angle a z b$.

Proof: Let $a, b$ with $\|a\| \geq\|b\|$ denote the two vertices of $L_{u v}$. If the segment $a z$ does not intersect $\partial \mathbb{D}$, the triangle auv is contained in $\mathbb{D}$ and with area $\frac{1}{2}\|u-v\|\|z-a\|=$ $\frac{1}{2}(2 r)(\sqrt{3} r)=r(\sqrt{3} r) \geq r t$. Otherwise, let $c$ denote the intersection point of $a z$ and $\partial \mathbb{D}$. See Fig. 5. Note that $t$ is the shortest distance from $z$ to $\partial \mathbb{D}$. The triangle $c u v$ is contained in $\mathbb{D}$ and with area $\frac{1}{2}\|u-v\|\|z-c\|=\frac{1}{2}(2 r)\|z-c\|=$ $r\|z-c\| \geq r t$. In addition, the half lens surrounded by the segment $u v$ and arcs $u b$ and $v b$ is always contained in $\mathbb{D}$. So, we have

$$
\left|L_{u v} \cap \mathbb{D}\right| \geq \frac{1}{2}\left|L_{u v}\right|+r t,
$$

and the lemma is proved.

For $z \in \mathbb{D}$ and $r \in \mathbb{R}$, let $\theta(z, r)$ denote the (total) central angle corresponding to the portion of $\partial B(z, r)$ in which if a diameter of $B(z, r)$ has an endpoint, the diameter is fully contained in $\mathbb{D}$. For example, in Figure (6), $b, c$ are the intersection points of $\partial B(z, r)$ and $\partial \mathbb{D}$, and the segment $b d$ is a diameter of $B(z, r)$. Then, $\theta(z, r)=4 \angle a z b$. In addition, if $z \in \mathbb{D}_{r}(1)$, we use $t(z)$ to denote the distance between $z$ and $\partial \mathbb{D}$. We have

$$
\begin{aligned}
& \theta(z, r)=2 \pi, \text { if } z \in \mathbb{D}_{r}(0) ; \\
& \theta(z, r) \leq 4 \arcsin \frac{t(z)}{r} \leq 4 \frac{t(z)}{r}, \text { if } z \in \mathbb{D}_{r}(1) ; \\
& \theta(z, r)=0, \text { if } z \in \mathbb{D}_{r}(2) .
\end{aligned}
$$

Proof of Lemma 6: Let $z=\frac{u+v}{2}$ and $r=\frac{\|u-v\|}{2}$. If $z \in \mathbb{D}_{\sqrt{3} r}(0), L_{u v}$ is fully contained in $\mathbb{D}$ and

$$
\left|L_{u v} \cap \mathbb{D}\right|=\frac{1}{{\beta_{0}}^{2}} \pi\|u-v\|^{2}=\frac{4}{{\beta_{0}}^{2}} \pi r^{2} .
$$


First, we calculate the integration over $z \in \mathbb{D}_{\sqrt{3} r}(0)$.

$$
\begin{aligned}
& \frac{n^{2}}{2} \iint_{\substack{u+v \\
\frac{u, v \in \mathbb{D}}{2} \in \mathbb{D} \sqrt{3} r \\
r_{\xi} \leq\|u-v\|<R_{n}}} e^{-n\left|L_{u v} \cap \mathbb{D}\right|} d u d v \\
& =\frac{n^{2}}{2} \int_{r=\frac{r_{\xi}}{2}}^{\frac{R_{n}}{2}} \int_{z \in \mathbb{D}_{\sqrt{3} r}(0)} e^{-\frac{4}{\beta_{0}^{2}} n \pi r^{2}} 8 \pi r d z d r \\
& \sim 2 n^{2} \int_{r=\frac{r_{\xi}}{2}}^{\frac{R_{n}}{2}} e^{-\frac{4}{\beta_{0}^{2}} n \pi r^{2}} 2 \pi r d r \\
& =2 n^{2} \int_{r=\frac{r_{\xi}}{2}}^{\frac{R_{n}}{2}} e^{-\frac{4 n}{\beta_{0}{ }^{2}} \pi r^{2}} d\left(\pi r^{2}\right) \\
& =2 n^{2}\left(-\left.\frac{\beta_{0}^{2}}{4 n} e^{-n \pi r^{2}}\right|_{r=\frac{r_{\xi}}{2}} ^{\frac{R_{n}}{2}}\right) \\
& \sim \frac{\beta_{0}^{2}}{2} e^{-\xi} \text {. }
\end{aligned}
$$

Next, we calculate the integration over $z \in \mathbb{D}_{\sqrt{3} r}(1) \backslash \mathbb{D}_{r}(2)$. Let $t$ denote the distance from $z$ to $\partial \mathbb{D}$. By Lemma 9 and Eq. (6), we have $\left|L_{u v} \cap \mathbb{D}\right| \geq \frac{2}{\beta_{0}^{2}} \pi r^{2}+c_{1} r t$ and $r \theta(z, r) \leq c_{2} t$. Thus,

$$
\begin{aligned}
& \frac{n^{2}}{2} \iint_{\substack{u+v \\
\frac{u \in \mathbb{N}}{2} \in \mathbb{3}^{r}(1) \backslash \mathbb{D}_{r}(2) \\
r_{\xi} \leq\|u-v\|<R_{n}}} e^{-n\left|L_{u v} \cap \mathbb{D}\right|} d u d v
\end{aligned}
$$

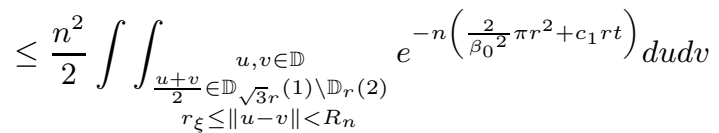

$$
\begin{aligned}
& \leq \frac{n^{2}}{2} \int_{r=\frac{r_{\xi}}{2}}^{\frac{R_{n}}{2}} \int_{z \in \mathbb{D}_{\sqrt{3} r}(1) \backslash \mathbb{D}_{r}(2)} e^{-n\left(\frac{2}{\beta_{0}{ }^{2}} \pi r^{2}+c_{1} r t\right)} 4 r \theta(z, r) \\
& \text { - } d z d r \\
& \leq O(1) n^{2} \int_{r=\frac{r_{\xi}}{2}}^{\frac{R_{n}}{2}} \int_{t=0}^{\sqrt{3} r} e^{-n\left(\frac{2}{\beta_{0}^{2}} \pi r^{2}+c_{1} r t\right)} t d t d r \\
& \leq O(1) n^{2} e^{-\frac{1}{2 \beta_{0}{ }^{2}} n \pi r_{\xi}^{2}} \int_{r=\frac{r_{\xi}}{2}}^{\frac{R_{n}}{2}} \int_{t=0}^{\sqrt{3} r} e^{-c_{1} n r t} t d t d r \\
& \leq O(1) n^{2} e^{-\frac{1}{2}(\ln n+\xi)} \int_{r=\frac{r_{\xi}}{2}}^{\frac{R_{n}}{2}} \int_{t=0}^{\infty} e^{-c_{1} n r t} t d t d r \\
& =O(1) n^{2} e^{-\frac{1}{2}(\ln n+\xi)} \int_{r=\frac{r_{\xi}}{2}}^{\frac{R_{n}}{2}}(n r)^{-2} d r \\
& \leq O(1) n^{2} e^{-\frac{1}{2}(\ln n+\xi)}\left(n r_{\xi}\right)^{-2} R_{n} \\
& \leq O(1) e^{-\frac{1}{2}(\ln n+\xi)}\left(\sqrt{\frac{\ln n}{n}}\right)^{-1}=O(1)(\ln n)^{-1 / 2} \\
& =o(1) .
\end{aligned}
$$

Last, we calculate the integration over $z \in \mathbb{D}_{r}(2)$. Since the measure of the set $\left\{(u, v) \mid u, v \in \mathbb{D}, \frac{u+v}{2} \in \mathbb{D}_{r}(2)\right\}$ is zero,

$$
\frac{n^{2}}{2} \iint_{\substack{u, v \in \mathbb{D} \\ \frac{u+v}{2} \in \mathbb{D}_{r}(2) \\ r_{\xi} \leq\|u-v\|<R_{n}}} e^{-n\left|L_{u v} \cap \mathbb{D}\right|} d u d v=0 .
$$

Therefore,

$$
\frac{n^{2}}{2} \iint_{\substack{u, v \in \mathbb{D} \\ r_{\xi} \leq\|u-v\|<R_{n}}} e^{-n\left|L_{u v} \cap \mathbb{D}\right|} d u d v \sim \frac{\beta_{0}{ }^{2}}{2} e^{-\xi} .
$$

\section{APPENDIX B \\ SupPlements to The Proof of Lemma 8}

To prove Lemma 8 , we introduce several relevant events and derive their probabilities. Let $A$ denote the disk centered at the origin with area $\frac{\ln n}{4 n}$, i.e. with radius $\frac{1}{2} \sqrt{\frac{\ln n}{n \pi}}$. Let $\beta_{1}$ and $\beta_{2}$ denote two positive constants, and $R_{1}$ and $R_{2}$ be given by $n \pi R_{1}^{2}=\beta_{1} \ln n$ and $n \pi R_{2}^{2}=\beta_{2} \ln n$. Choose $\beta_{1}, \beta_{2}$ such that $\max \left(\frac{1}{4} \beta_{0}^{2}, \beta^{2}\right)<\beta_{1}<\beta_{2}<\beta_{0}^{2}$ and $\frac{\pi}{c^{2}}\left(1-\frac{R_{1}}{R_{2}}\right)<1$. Here $c$ is given by Lemma 3. We have $\frac{1}{2} R_{2} \leq R_{1} \leq R_{2}$.

Assume $V$ is a point set and $Y \subset V$. Let $h_{1}(Y, V)$ denote a function such that $h_{1}(Y=\{u, v\}, V)=1$ only if $\frac{1}{2}(u+v) \in A, R_{1} \leq\|u-v\| \leq R_{2}$, and there is no other node of $V$ in $L_{u v}$; otherwise, $h_{1}(Y, V)=0$. Then, $E_{1}$ is the event that there exist two nodes $X, Y \in \mathcal{P}_{n}$ such that $h_{1}\left(\{X, Y\}, \mathcal{P}_{n}\right)=1$. In what follows, we use $X_{1}, X_{2}, X_{3}$ and $X_{4}$ to denote independent random points with uniform distribution over $\mathbb{D}$ and independent of $\mathcal{P}_{n}$. Let $F_{1}$ be the event that

$$
h_{1}\left(\left\{X_{1}, X_{2}\right\},\left\{X_{1}, X_{2}\right\} \cup \mathcal{P}_{n}\right)=1,
$$

$F_{2}$ be the event that

$$
\begin{aligned}
& h_{1}\left(\left\{X_{1}, X_{2}\right\},\left\{X_{1}, X_{2}, X_{3}\right\} \cup \mathcal{P}_{n}\right) \\
& \cdot h_{1}\left(\left\{X_{1}, X_{3}\right\},\left\{X_{1}, X_{2}, X_{3}\right\} \cup \mathcal{P}_{n}\right)=1,
\end{aligned}
$$

and $F_{3}$ be the event that

$$
\begin{aligned}
& h_{1}\left(\left\{X_{1}, X_{2}\right\},\left\{X_{1}, X_{2}, X_{3}, X_{4}\right\} \cup \mathcal{P}_{n}\right) \\
& \cdot h_{1}\left(\left\{X_{3}, X_{4}\right\},\left\{X_{1}, X_{2}, X_{3}, X_{4}\right\} \cup \mathcal{P}_{n}\right)=1 .
\end{aligned}
$$

We claim that

$$
\operatorname{Pr}\left[E_{1}\right] \geq \frac{n^{2}}{2} \operatorname{Pr}\left[F_{1}\right]-\frac{n^{3}}{2} \operatorname{Pr}\left[F_{2}\right]-\frac{n^{4}}{8} \operatorname{Pr}\left[F_{3}\right] .
$$

We shall prove this claim by the Palm theory and Boole's inequalities. For clarity, we use $X_{1}^{\prime}, X_{2}^{\prime}, X_{3}^{\prime}$ and $X_{4}^{\prime}$ to denote elements of $\mathcal{P}_{n}$. For any $\left\{x_{1}, x_{2}, x_{3}\right\} \subseteq V$, let

$$
\begin{aligned}
h_{2}\left(\left\{x_{1}, x_{2}, x_{3}\right\}, V\right) & =h_{1}\left(\left\{x_{1}, x_{2}\right\}, V\right) \cdot h_{1}\left(\left\{x_{1}, x_{3}\right\}, V\right) \\
& +h_{1}\left(\left\{x_{2}, x_{1}\right\}, V\right) \cdot h_{1}\left(\left\{x_{2}, x_{3}\right\}, V\right) \\
& +h_{1}\left(\left\{x_{3}, x_{1}\right\}, V\right) \cdot h_{1}\left(\left\{x_{3}, x_{2}\right\}, V\right) .
\end{aligned}
$$

For any $\left\{x_{1}, x_{2}, x_{3}, x_{4}\right\} \subseteq V$, let

$$
\begin{aligned}
& h_{3}\left(\left\{x_{1}, x_{2}, x_{3}, x_{4}\right\}, V\right) \\
& =h_{1}\left(\left\{x_{1}, x_{2}\right\}, V\right) \cdot h_{1}\left(\left\{x_{3}, x_{4}\right\}, V\right) \\
& +h_{1}\left(\left\{x_{1}, x_{3}\right\}, V\right) \cdot h_{1}\left(\left\{x_{2}, x_{4}\right\}, V\right) \\
& +h_{1}\left(\left\{x_{1}, x_{4}\right\}, V\right) \cdot h_{1}\left(\left\{x_{2}, x_{3}\right\}, V\right) .
\end{aligned}
$$

Note that the addition and multiplication in $h_{2}$ and $h_{3}$ are Boolean operators. Let $F_{1}^{\prime}\left(\left\{X_{1}^{\prime}, X_{2}^{\prime}\right\}\right)$ be the events that $h_{1}\left(\left\{X_{1}^{\prime}, X_{2}^{\prime}\right\}, \mathcal{P}_{n}\right)=1, F_{2}^{\prime}\left(\left\{X_{1}^{\prime}, X_{2}^{\prime}, X_{3}^{\prime}\right\}\right)$ be the event that $h_{2}\left(\left\{X_{1}^{\prime}, X_{2}^{\prime}, X_{3}^{\prime}\right\}, \mathcal{P}_{n}\right)=1$, and $F_{3}^{\prime}\left(\left\{X_{1}^{\prime}, X_{2}^{\prime}, X_{3}^{\prime}, X_{4}^{\prime}\right\}\right)$ be the events that $h_{3}\left(\left\{X_{1}^{\prime}, X_{2}^{\prime}, X_{3}^{\prime}, X_{4}^{\prime}\right\}, \mathcal{P}_{n}\right)=1$. According to 
the Palm theory (refer to Theorem 7 in [14]), we have

$$
\begin{aligned}
& \sum_{\left\{X_{1}^{\prime}, X_{2}^{\prime}\right\} \subseteq \mathcal{P}_{n}} \operatorname{Pr}\left[F_{1}^{\prime}\left(\left\{X_{1}^{\prime}, X_{2}^{\prime}\right\}\right)\right] \\
& =\mathbf{E}\left[\sum_{\left\{X_{1}^{\prime}, X_{2}^{\prime}\right\} \subseteq \mathcal{P}_{n}} h_{1}\left(\left\{X_{1}^{\prime}, X_{2}^{\prime}\right\}, \mathcal{P}_{n}\right)\right] \\
& =\frac{n^{2}}{2 !} \mathbf{E}\left[h_{1}\left(\left\{X_{1}, X_{2}\right\},\left\{X_{1}, X_{2}\right\} \cup \mathcal{P}_{n}\right)\right] \\
& =\frac{n^{2}}{2} \operatorname{Pr}\left[F_{1}\right] \text {; } \\
& \sum_{\left\{X_{1}^{\prime}, X_{2}^{\prime}, X_{3}^{\prime}\right\} \subseteq \mathcal{P}_{n}} \operatorname{Pr}\left[F_{2}^{\prime}\left(\left\{X_{1}^{\prime}, X_{2}^{\prime}, X_{3}^{\prime}\right\}\right)\right] \\
& =\mathbf{E}\left[\sum_{\left\{X_{1}^{\prime}, X_{2}^{\prime}, X_{3}^{\prime}\right\} \subseteq \mathcal{P}_{n}} h_{2}\left(\left\{X_{1}^{\prime}, X_{2}^{\prime}, X_{3}^{\prime}\right\}, \mathcal{P}_{n}\right)\right] \\
& =\frac{n^{3}}{3 !} \mathbf{E}\left[h_{2}\left(\left\{X_{1}, X_{2}, X_{3}\right\},\left\{X_{1}, X_{2}, X_{3}\right\} \cup \mathcal{P}_{n}\right)\right] \\
& =3 \frac{n^{3}}{3 !} \operatorname{Pr}\left[F_{2}\right]=\frac{n^{3}}{2} \operatorname{Pr}\left[F_{2}\right] \text {; }
\end{aligned}
$$

and

$$
\begin{aligned}
& \sum_{\left\{X_{1}^{\prime}, X_{2}^{\prime}, X_{3}^{\prime}, X_{4}^{\prime}\right\} \subseteq \mathcal{P}_{n}} \operatorname{Pr}\left[F_{3}^{\prime}\left(\left\{X_{1}^{\prime}, X_{2}^{\prime}, X_{3}^{\prime}, X_{4}^{\prime}\right\}\right)\right] \\
= & \mathbf{E}\left[\sum_{\left\{X_{1}^{\prime}, X_{2}^{\prime}, X_{3}^{\prime}, X_{4}^{\prime}\right\} \subseteq \mathcal{P}_{n}} h_{3}\left(\left\{X_{1}^{\prime}, X_{2}^{\prime}, X_{3}^{\prime}, X_{4}^{\prime}\right\}, \mathcal{P}_{n}\right)\right] \\
= & \frac{n^{4}}{4 !} \mathbf{E}\left[h_{3}\left(\left\{X_{1}, X_{2}, X_{3}, X_{4}\right\},\left\{X_{1}, X_{2}, X_{3}, X_{4}\right\} \cup \mathcal{P}_{n}\right)\right] \\
= & 3 \frac{n^{4}}{4 !} \operatorname{Pr}\left[F_{3}\right]=\frac{n^{4}}{8} \operatorname{Pr}\left[F_{3}\right] .
\end{aligned}
$$

Applying Boole's inequalities and Eq. (8), (9), and (10), we have

$$
\begin{aligned}
\operatorname{Pr}\left[E_{1}\right] \geq & \sum_{\left\{X_{1}^{\prime}, X_{2}^{\prime}\right\} \subseteq \mathcal{P}_{n}} \operatorname{Pr}\left[F_{1}^{\prime}\left(\left\{X_{1}^{\prime}, X_{2}^{\prime}\right\}\right)\right] \\
- & \sum_{\left\{X_{1}^{\prime}, X_{2}^{\prime}, X_{3}^{\prime}\right\} \subseteq \mathcal{P}_{n}} \operatorname{Pr}\left[F_{2}^{\prime}\left(\left\{X_{1}^{\prime}, X_{2}^{\prime}, X_{3}^{\prime}\right\}\right)\right] \\
& -\sum_{\left\{X_{1}^{\prime}, X_{2}^{\prime}, X_{3}^{\prime}, X_{4}^{\prime}\right\} \subseteq \mathcal{P}_{n}} \operatorname{Pr}\left[F_{3}^{\prime}\left(\left\{X_{1}^{\prime}, X_{2}^{\prime}, X_{3}^{\prime}, X_{4}^{\prime}\right\}\right)\right] \\
= & \frac{n^{2}}{2} \operatorname{Pr}\left[F_{1}\right]-\frac{n^{3}}{2} \operatorname{Pr}\left[F_{2}\right]-\frac{n^{4}}{8} \operatorname{Pr}\left[F_{3}\right] .
\end{aligned}
$$

Hence, our claim is true.

In the next, we derive the probabilities of $F_{1}, F_{2}$, and $F_{3}$. Let $S_{1}$ denote the set

$$
\left\{\left(x_{1}, x_{2}\right) \mid \frac{1}{2}\left(x_{1}+x_{2}\right) \in A, R_{1} \leq\left\|x_{1}-x_{2}\right\| \leq R_{2}\right\} .
$$

We have

$$
\begin{aligned}
\operatorname{Pr}\left[F_{1}\right] & =\iint_{S_{1}} \operatorname{Pr}\left[F_{1} \mid X_{1}=x_{1}, X_{2}=x_{2}\right] d x_{1} d x_{2} \\
& =\iint_{S_{1}} e^{-n\left|L_{x_{1}} x_{2}\right|} d x_{1} d x_{2} \\
& =\iint_{S_{1}} e^{-n \frac{1}{\beta_{0}^{2}} \pi\left\|x_{1-x_{2}}\right\|^{2}} d x_{1} d x_{2} .
\end{aligned}
$$

Let $z=\frac{x_{1}+x_{2}}{2}$ and $r=\frac{1}{2}\left\|x_{1}-x_{2}\right\|$. Then,

$$
\begin{aligned}
\operatorname{Pr}\left[F_{1}\right] & =\int_{z \in A} \int_{r=\frac{R_{1}}{2}}^{\frac{R_{2}}{2}} e^{-\frac{4}{\beta_{0}^{2}} n \pi r^{2}} 8 \pi r d r d z \\
& =4 \int_{z \in A} \int_{r=\frac{R_{1}}{2}}^{\frac{R_{2}}{2}} e^{-\frac{4}{\beta_{0}^{2}} n \pi r^{2}} 2 \pi r d r d z \\
& =4 \int_{z \in A} \int_{r=\frac{R_{1}}{2}}^{\frac{R_{2}}{2}} e^{-\frac{4}{\beta_{0}^{2}} n \pi r^{2}} d\left(\pi r^{2}\right) d z \\
& =-\left(\left.\frac{\beta_{0}^{2}}{n} e^{-\frac{4}{\beta_{0}^{2}} n \pi r^{2}}\right|_{r=\frac{R_{1}}{2}} ^{\frac{R_{2}}{2}}\right)|A| \\
& =\frac{\beta_{0}^{2}}{4 n^{2}}\left(n^{-\frac{\beta_{1}}{\beta_{0}{ }^{2}}}-n^{-\frac{\beta_{2}}{\beta_{0}^{2}}}\right) \ln n .
\end{aligned}
$$

Let $S_{2}$ denote the set

$$
\left\{\begin{array}{c|c}
\left(x_{1}, x_{2}, x_{3}\right) & \begin{array}{c}
\frac{x_{1}+x_{2}}{2}, \frac{x_{1}+x_{3}}{2} \in A ; x_{1}, x_{2} \notin L_{x_{1} x_{3}} ; \\
x_{1}, x_{3} \notin L_{x_{1} x_{2}} ; R_{1} \leq\left\|x_{1}-x_{2}\right\| \leq R_{2} ; \\
R_{1} \leq\left\|x_{1}-x_{3}\right\| \leq R_{2}
\end{array}
\end{array}\right\} .
$$

Applying Lemma 3 , if $\left(x_{1}, x_{2}, x_{3}\right) \in S_{2}$, we have

$$
\begin{aligned}
& \operatorname{Pr}\left[F_{2} \mid X_{1}=x_{1}, X_{2}=x_{2}, X_{3}=x_{3}\right] \leq e^{-n\left|L_{x_{1} x_{2}} \cup L_{x_{1} x_{3}}\right|} \\
& \leq e^{-n\left(\frac{1}{\beta_{0}} \pi\left\|x_{1}-x_{2}\right\|^{2}+c R_{2}\left\|\frac{x_{1}+x_{2}}{2}-\frac{x_{1}+x_{3}}{2}\right\|\right)} .
\end{aligned}
$$

Therefore,

$$
\begin{aligned}
& \operatorname{Pr}\left[F_{2}\right] \\
& =\iiint_{S_{2}} \operatorname{Pr}\left[F_{2} \mid X_{1}=x_{1}, X_{2}=x_{2}, X_{3}=x_{3}\right] \\
& \quad \cdot d x_{1} d x_{2} d x_{3} \\
& \leq \iiint_{S_{2}} e^{-n\left(\frac{1}{\beta_{0}^{2}} \pi\left\|x_{1}-x_{2}\right\|^{2}+c R_{2}\left\|\frac{x_{1}+x_{2}}{2}-\frac{x_{1}+x_{3}}{2}\right\|\right)} \\
& \quad \cdot d x_{1} d x_{2} d x_{3} .
\end{aligned}
$$

Let $z_{1}=\frac{x_{1}+x_{2}}{2}, r_{1}=\frac{1}{2}\left\|x_{1}-x_{2}\right\|, z_{2}=\frac{x_{1}+x_{3}}{2}$, and $\rho=$ $\left\|z_{1}-z_{2}\right\|$. Then,

$$
\begin{aligned}
& \operatorname{Pr}\left[F_{2}\right] \\
& \leq 16 \int_{z_{1} \in A} \int_{r_{1}=\frac{R_{1}}{2}}^{\frac{R_{2}}{2}} \int_{z_{2} \in A} e^{-n\left(\frac{4}{\beta_{0} 2} \pi r_{1}^{2}+c R_{2}\left\|z_{1}-z_{2}\right\|\right)} 2 \pi r_{1} \\
& \\
& \quad \cdot d r_{1} d z_{1} d z_{2} \\
& \leq 16 \int_{z_{1} \in A} \int_{r_{1}=\frac{R_{1}}{2}}^{\frac{R_{2}}{2}} e^{-\frac{4}{\beta_{0}^{2}} n \pi r_{1}^{2}} 2 \pi r_{1} d r_{1} d z_{1} \\
& \quad \int_{z_{2} \in A} e^{-c n R_{2}\left\|z_{1}-z_{2}\right\|} d z_{2} \\
& \leq 16 \int_{z_{1} \in A} \int_{r_{1}=\frac{R_{1}}{2}}^{\frac{R_{2}}{2}} e^{-\frac{4}{\beta_{0}^{2}} n \pi r_{1}^{2}} d\left(\pi r_{1}^{2}\right) d z_{1}
\end{aligned}
$$




$$
\begin{aligned}
& \cdot \int_{\rho=0}^{\infty} e^{-c n R_{2} \rho} 2 \pi \rho d \rho \\
=- & \left(\left.\frac{4 \beta_{0}^{2}}{n} e^{-\frac{4}{\beta_{0}^{2}} n \pi r^{2}}\right|_{r=\frac{R_{1}}{2}} ^{\frac{R_{2}}{2}}\right)|A| \frac{2 \pi}{\left(c n R_{2}\right)^{2}} \\
= & \frac{2 \pi \beta_{0}^{2}}{c^{2}\left(n R_{2}^{2}\right) n^{3}}\left(n^{-\frac{\beta_{1}}{\beta_{0}^{2}}}-n^{-\frac{\beta_{2}}{\beta_{0}^{2}}}\right) \ln n .
\end{aligned}
$$

Let $S_{3}$ denote the set

$$
\left\{\begin{array}{c|c}
\left(x_{1}, x_{2}, x_{3}, x_{4}\right) & \frac{x_{1}+x_{2}}{2}, \frac{x_{3}+x_{4}}{2} \in A ; \\
x_{1}, x_{2} \notin L_{x_{3} x_{4}} ; x_{3}, x_{4} \notin L_{x_{1} x_{2}} ; \\
R_{1} \leq\left\|x_{1}-x_{2}\right\| \leq R_{2} ; \\
R_{1} \leq\left\|x_{3}-x_{4}\right\| \leq R_{2}
\end{array}\right\} .
$$

Applying Lemma 3 , if $\left(x_{1}, x_{2}, x_{3}, x_{4}\right) \in S_{3}$, we have

$$
\begin{aligned}
& \operatorname{Pr}\left[F_{3} \mid X_{1}=x_{1}, X_{2}=x_{2}, X_{3}=x_{3}, X_{4}=x_{4}\right] \\
& \leq e^{-n\left|L_{x_{1} x_{2}} \cup L_{x_{3} x_{4}}\right|} \\
& \leq e^{-n\left(\frac{1}{\beta_{0}^{2}} \pi\left\|x_{1}-x_{2}\right\|^{2}+c R_{2}\left\|\frac{x_{1}+x_{2}}{2}-\frac{x_{3}+x_{4}}{2}\right\|\right)} .
\end{aligned}
$$

Therefore,

$$
\begin{aligned}
& \operatorname{Pr}\left[F_{3}\right] \\
& =\iiint \int_{S_{3}} \operatorname{Pr}\left[F_{3} \mid \begin{array}{c}
X_{1}=x_{1}, X_{2}=x_{2}, \\
X_{3}=x_{3}, X_{4}=x_{4}
\end{array}\right] \\
& \quad \cdot d x_{1} d x_{2} d x_{3} d x_{4} \\
& \leq \iiint \int_{S_{3}} e^{-n\left(\frac{1}{\beta_{0}^{2}} \pi\left\|x_{1}-x_{2}\right\|^{2}+c R_{2}\left\|\frac{x_{1}+x_{2}}{2}-\frac{x_{3}+x_{4}}{2}\right\|\right)} \\
& \quad \cdot d x_{1} d x_{2} d x_{3} d x_{4} .
\end{aligned}
$$

Let $z_{1}=\frac{x_{1}+x_{2}}{2}, r_{1}=\frac{1}{2}\left\|x_{1}-x_{2}\right\|, z_{2}=\frac{x_{3}+x_{4}}{2}, r_{2}=$ $\frac{1}{2}\left\|x_{3}-x_{4}\right\|$, and $\rho=\left\|z_{1}-z_{2}\right\|$. Then,

$$
\begin{aligned}
\operatorname{Pr} & {\left[F_{3}\right] } \\
\leq & \int_{z_{1} \in A} \int_{r_{1}=\frac{R_{1}}{2}}^{\frac{R_{2}}{2}} \int_{z_{2} \in A} \int_{r_{2}=\frac{R_{1}}{2}}^{\frac{R_{2}}{2}} e^{-n\left(\frac{4}{\beta_{0}^{2}} \pi r_{1}^{2}+c R_{2}\left\|z_{1}-z_{2}\right\|\right)} \\
& \cdot\left(8 \pi r_{1} d r_{1} d z_{1}\right)\left(8 \pi r_{2} d r_{2} d z_{2}\right) \\
\leq & \left(4 \int_{z_{1} \in A} \int_{r_{1}=\frac{R_{1}}{2}}^{\frac{R_{2}}{2}} e^{-\frac{4}{\beta_{0}^{2}} n \pi r_{1}^{2}} 2 \pi d r_{1} d z_{1}\right) \\
& \cdot\left(8 \pi \frac{R_{2}}{2}\left(\frac{R_{2}}{2}-\frac{R_{1}}{2}\right) \int_{z_{2} \in A} e^{-c n R_{2}\left\|z_{1}-z_{2}\right\|} d z_{2}\right) \\
\leq & \left(4 \int_{z_{1} \in A} \int_{r_{1}=\frac{R_{1}}{2}}^{\frac{R_{2}}{2}} e^{-\frac{4}{\beta_{0}^{2}} n \pi r_{1}^{2}} d\left(\pi r_{1}^{2}\right) d z_{1}\right) \\
& \cdot\left(8 \pi \frac{R_{2}}{2}\left(\frac{R_{2}}{2}-\frac{R_{1}}{2}\right) \int_{\rho=0}^{\infty} e^{-c n R_{2} \rho} 2 \pi \rho d \rho\right) \\
= & \left.\frac{\beta_{0}^{2} \ln n}{4 n^{2}}\left(n^{-\frac{\beta_{1}}{\beta_{0}{ }^{2}}}-n^{-\frac{\beta_{2}}{\beta_{0}{ }^{2}}}\right)\right) \\
& \cdot\left(\frac{4 \pi^{2}}{\left(c n R_{2}\right)^{2}} R_{2}\left(R_{2}-R_{1}\right)\right) \\
= & \frac{\pi^{2} \beta_{0}^{2}}{c^{2} n^{4}}\left(1-\frac{R_{1}}{R_{2}}\right)\left(n^{-\frac{\beta_{1}}{\beta_{0}^{2}}}-n^{-\frac{\beta_{2}}{\beta_{0}{ }^{2}}}\right) \ln n .
\end{aligned}
$$

Put Eq. (7), (11), (12) and (13) together. We have

$$
\begin{aligned}
& \operatorname{Pr}\left[E_{1}\right] \\
& \geq\left(\frac{\beta_{0}{ }^{2}}{8}-\frac{\pi \beta_{0}^{2}}{c^{2}\left(n R_{2}^{2}\right)}-\frac{\pi^{2} \beta_{0}{ }^{2}}{8 c^{2}}\left(1-\frac{R_{1}}{R_{2}}\right)\right) \\
& \quad \cdot\left(n^{-\frac{\beta_{1}}{\beta_{0}^{2}}}-n^{-\frac{\beta_{2}}{\beta_{0}^{2}}}\right) \ln n \\
& \sim \frac{\beta_{0}{ }^{2}}{8}\left(1-\frac{\pi^{2}}{c^{2}}\left(1-\frac{R_{1}}{R_{2}}\right)\right)\left(n^{-\frac{\beta_{1}}{\beta_{0}^{2}}}-n^{-\frac{\beta_{2}}{\beta_{0}^{2}}}\right) \ln n .
\end{aligned}
$$

Since $\frac{\pi^{2}}{c^{2}}\left(1-\frac{R_{1}}{R_{2}}\right)<1$ and $I_{n}=\Omega\left(\frac{n}{\ln n}\right)$, we have

$$
\operatorname{Pr}\left[E_{1}\right]=\Omega\left(\left(n^{-\frac{\beta_{1}}{\beta_{0}^{2}}}-n^{-\frac{\beta_{2}}{\beta_{0}^{2}}}\right) \ln n\right),
$$

and

$$
I_{n} \operatorname{Pr}\left[E_{1}\right]=\Omega\left(n^{1-\frac{\beta_{1}}{\beta_{0}}}\right) \rightarrow \infty
$$

\section{ACKNOWLEDGMENT}

This work of Dr. Yi described in this paper was partially supported by NSC under Grant No. NSC97-2221-E-009-052MY3, by MoEA under Grant No. 98-EC-17-A-02-S2-0048, by ITRI under Grant No. 8352BA4124, and by the MoE ATU plan.

The work of Dr. Wan described in this paper was partially supported by NSF of USA under grant CNS-0831831.

\section{REFERENCES}

[1] M. Penrose, Random Geometric Graphs. Oxford University Press, 2003.

[2] E. N. Gilbert, "Random plane networks," J. Society Industrial Applied Mathematics, vol. 9, no. 4, pp. 533-543, Dec. 1961.

[3] B. Karp and H. Kung, "GPSR: greedy perimeter stateless routing for wireless networks," in Proc. 6th Annual International Conf. Mobile Computing Netw. (MobiCom '00), Aug. 2000, pp. 243-254.

[4] P. Bose, P. Morin, I. Stojmenović, and J. Urrutia, "Routing with guaranteed delivery in ad hoc wireless networks," Wireless Netw., vol. 7, no. 6, pp. 609-616, Nov. 2001.

[5] G. T. Toussaint, "The relative neighbourhood graph of a finite planar set," Pattern Recognition, vol. 12, no. 4, pp. 261-268, 1980.

[6] H. Dette and N. Henze, "The limit distribution of the largest nearestneighbour link in the unit $d$-cube," J. Applied Probability, vol. 26, no. 1, pp. 67-80, 1989.

[7] T. K. Philips, S. S. Panwar, and A. N. Tantawi, "Connectivity properties of a packet network radio model," IEEE Trans. Inf. Theory, vol. 35, no. 5, pp. 1044-1047, Sept. 1989.

[8] M. D. Penrose, "The longest edge of the random minimal spanning tree," Annals Applied Probability, vol. 7, no. 2, pp. 340-361, May 1997.

[9] P. Santi and D. M. Blough, "The critical transmitting range for connectivity in sparse wireless ad hoc networks," IEEE Trans. Mobile Computing, vol. 2, no. 1, pp. 25-39, Jan.-Mar. 2003.

[10] G. Kozma, Z. Lotker, M. Sharir, and G. Stupp, "Geometrically aware communication in random wireless networks," in Proc. Twenty-Third Annual ACM Symp. Principles Distributed Computing, July 2004, pp. 310-319.

[11] A. Goel, S. Rai, and B. Krishnamachari, "Sharp thresholds for monotone properties in random geometric graphs," in Proc. 36th ACM Symp. Theory Computing (STOC 2004), June 2004, pp. 580-586.

[12] P. Gupta and P. R. Kumar, "Critical power for asymptotic connectivity in wireless networks," in Stochastic Analysis, Control, Optimization Applications: A Volume in Honor of W. H. Fleming, W. M. McEneaney, G. Yin, and Q. Zhang, Eds. Birkhauser, Mar. 1998, pp. 547-566.

[13] P.-J. Wan and C.-W. Yi, "Asymptotic critical transmission ranges for connectivity in wireless ad hoc networks with Bernoulli nodes," in IEEE Wireless Commun. Netw. Conf. (WCNC 2005), Mar. 2005.

[14] P.-J. Wan, C.-W. Yi, F. Yao, and X. Jia, "Asymptotic critical transmission radius for greedy forward routing in wireless ad hoc networks," in Proc. 7th ACM International Symp. Mobile Ad Hoc Netw. Computing, May 2006, pp. 25-36. 
[15] P. Bose, L. Devroye, W. Evans, and D. Kirkpatrick, "On the spanning ratio of gabriel graphs and beta-skeletons," in Proc. Latin American Theoretical Infocomatics (LATIN), 2002.

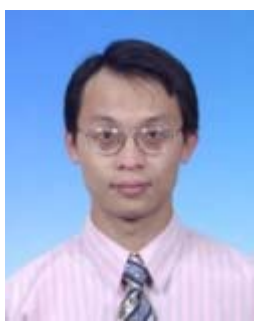

Chih-Wei Yi Dr. Chih-Wei Yi received his $\mathrm{PhD}$ degree from the Illinois Institute of Technology, and MS and BS degrees from the National Taiwan University. $\mathrm{He}$ is currently an Assistant Professor in Computer Science at the National Chiao Tung University. $\mathrm{He}$ is a member of the IEEE and the ACM. He had been a Senior Research Fellow of the Department of Computer Science, City University of Hong Kong. He was bestowed the Outstanding Young Engineer Award by the Chinese Institute of Engineers in 2009. His research focuses on wireless ad hoc and sensor networks, vehicular ad hoc networks, network coding, and algorithm design and analysis.

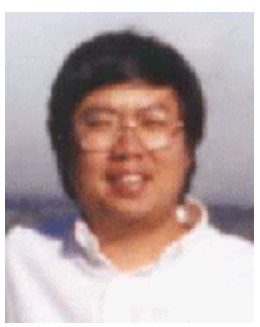

Peng-Jun Wan Dr. Peng-Jun Wan received his PhD degree from University of Minnesota, MS degree from The Chinese Academy of Science, and BS degree from Tsinghua University. He is currently an Associate Professor in Computer Science at Illinois Institute of Technology, and at City University of Hong Kong. His research interests include wireless networks, optical networks, and algorithm design and analysis.

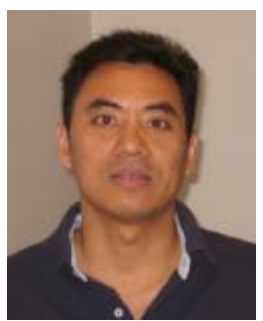

Lixin Wang Mr. Lixin Wang received the M.S. degree in CS from the University of Houston at Clear Lake, the M.S. degree in Applied Math from the University of Houston and the M.S. degree in Math from the Fudan University, Shanghai, China. $\mathrm{He}$ is currently a Ph.D. student in Computer Science at the Illinois Institute of Technology, Chicago. His research is on wireless networks, and algorithm design and analysis.

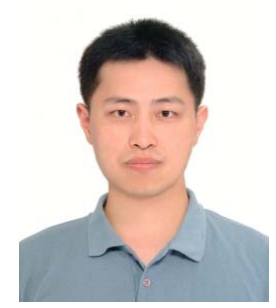

Chao-Min Su Mr. Chao-Min Su received his MS and BS degree from the National Taiwan University. Currently, he is a Ph.D. candidate in Computer Science at the National Chiao Tung University. His research interests are in wireless ad hoc and sensor networks, and sensor applications. 